\title{
Evaluación de los niveles de cortisol y testosterona durante el proceso de colecta de semen mediante electroeyaculador en carneros con y sin tranquilizante.
}

Evaluation of cortisol and testosterone levels during the semen collection process by electroejaculator in rams with and without tranquilizer.

Luis Alejandro Ulloa Ramones. ${ }^{1} \&$ Diego Alberto Ulloa Ramones. ${ }^{2}$

Recibido:01-02-2020 / Revisado: 25-02-2020 /Aceptado: 03-03-2020/ Publicado: 04-04-2020

\begin{abstract}
.
DOI: https://doi.org/10.33262/cienciadigital.v4i2.1206
\end{abstract}

The most commonly used techniques for collecting semen, either for conservation purposes of genetic or reproductive material are: the artificial vagina and the electro-ejaculator. There has been controversy over the use of the electroejaculator by the scientific community due to the stress caused by this method and it would be considered to be an attempt to animal welfare. The objective of this study was to assess the levels of stress caused by the use of the electro ejaculator in rams treated with tranquilizers and without tranquilizers. Cortisol and testosterone levels were measured in sexually mature rams of the Corriedale breed between 2-3 years of age, with weights of $60-90 \mathrm{~kg}$. 8 collects were made to the rams, where 4 collections were administered Xylazine (2\%) IV at a dose of $0.05 \mathrm{mg} / \mathrm{kg}$ live weight 10 minutes before the collection, and the remaining 4 collections were not administered tranquilizer. For the determination of the hormonal cortisol levels and testosterone blood samples were taken 20 minutes after using the electro ejaculator, the hormonal levels were measured by the ELISA test. For the results analysis, the Kolmogorov-Smirnov and ShapiroWilk tables were taken as reference for hormonal analyzes with and without Xylazine, as well as the Mann-Whitney parametric tests were used as a basis. The statistical tests showed

\footnotetext{
${ }^{1}$ Docente de la ESPOCH, Sede Morona Santiago, Macas, Ecuador, luis.alejandro86@ hotmail.com

${ }^{2}$ Médico Veterinario Zootecnista, Sucúa, Morona Santiago, Ecuador, diego_alberto_93@hotmail.com
} 
no significant difference in the use of the 2 treatments, resulting in similar levels of cortisol and testosterone in the treatments.

Keywords: Electroejaculator, Xylazine, Rams, Cortisol, Testosterone.

\section{Resumen.}

Las técnicas más utilizadas para la recolección se semen ya sea con fines de conservación de material génico o reproductivos son: la vagina artificial y el electro eyaculador. Ha existido controversia por el uso del electroeyaculador por la comunidad científica debido al estrés que causa este método y se consideraría atentar al bienestar animal. El objetivo de este estudio fue valorar los niveles de estrés causados por el uso del electro eyaculador en carneros tratados con tranquilizantes y sin tranquilizante. Se midió los niveles de cortisol y testosterona en carneros maduros sexualmente de la raza Corriedale los mismos que estaban en un promedio de 2,5 años \pm 0.5 años, con un peso promedio de $75 \mathrm{~kg} \pm 15 \mathrm{~kg}$ de los semovientes. El número de colectas que se realizaron a los carneros fueron de 8 , administrándose a las 4 primeras colectas Xilacina en concentración al $2 \%$ mediante IV a dosis de $0.05 \mathrm{mg} / \mathrm{kg}, 10$ minutos antes de realizar el procedimiento de colecta, las 4 últimas colectas no se realizaron la aplicación tranquilizante. Para la determinación de los niveles hormonales de cortisol y testosterona se produjo a extraer muestras de sangre 20 minutos después del uso del electro eyaculador, los niveles hormonales se midieron mediante la prueba de ELISA. Para el análisis de resultados se tomó como referencia las tablas de Kolmogorov-Smirnov y Shapiro-Wilk para los análisis hormonales con y sin Xilacina, así mismo se utilizó como base las pruebas paramétricas de Mann-Whitney Las pruebas estadísticas no mostraron diferencia significativa en el uso de los 2 tratamientos, dando como resultado niveles similares de cortisol y testosterona en los tratamientos.

Palabras claves: Electroeyaculador, Xilacina, Carneros, Cortisol, Testosterona.

\section{Introducción.}

Con el avance de la ciencia, se ha buscado maneras para la preservación de material genético para luego ser usados, como en animales de alto valor genético, o en problemas de extinción u otros, con el fin de conservar y prolongar la herencia de animales considerados como importantes y uno de los métodos usados es la extracción de semen en machos, especialmente en animales de alto valor genético que no estén en condiciones de monta, con problemas estructurales o tengan problemas reproductivos por accidentes o longevos, de esta manera se han desarrollado varios métodos y alternativas para la extracción de seminal.

Las técnicas comúnmente usadas para la colecta seminal en carnero son: vagina artificial, electro eyaculador y el masaje transrectal de las glándulas sexuales accesorias, en consecuencia, al usar el electro eyaculador suele producir estrés en los animales, lo que se podría considerar como un atentado al bienestar animal, esto viene acarreando inconvenientes por la problemática de que se 
viola los estándares de estrés en animales, teniendo como inconveniente el aplicar este método como un sistema efectivo para la colección seminal en animales.

El uso de la vagina artificial es uno de los métodos que menos estrés causa, y con una mejor calidad de semen en la colecta por su similitud fisiológica y su nivel de estrés muy bajo (Sylla, Palombi, Stradaioli, Vagniluca, \& Monaci, 2015); pero la el inconveniente principal de esta técnica es que se necesita de instalaciones adecuadas, animales no nerviosos y que hayan tenido une entrenamiento previo, con esto se corroboraría que el uso de la vagina artificial tomaría como mejor técnica para el uso ya que se evitaría estrés y tendría un manejo similar al natural, pero esto caería en disputa si los animales en los cuales se requiere obtener material génico tienen dificultades en la monta, problemas en los miembros locomotores o situaciones en las cuales no permita la extracción del contenido seminal con vagina artificial ni con masajes de las glándulas sexuales accesorias.

La electroeyaculación ( EE) es un método de colección seminal utilizada en machos que tienen relevancia genética o para su criopreservarción, este método se aplica en carneros que no han sido entrenados con vagina artificial, que tienen problemas de monta (podales o de miembros locomotores, problemas en la columna o por falta de libido) o por problemas eyaculatorios.

En varios estudios se afirma que el método de la electroeyaculación es un método eficaz para la recolección de semen (Palmer, Brito, Soderquist, Persson, \& Barth, 2005), pero en varios países está prohibida esta técnica si el animal no está debidamente anestesiados o sedado por el posible dolor que causa, para ello se realizan varios estudios para la comprobación de la técnica mejor adecuada para evitar el maltrato hacia los animales, pero no se debe dejar de lado cual es la técnica más eficiente en relación a la calidad, cantidad y viabilidad espermática.

La colecta seminal por este método son de menor calidad que la obtenida con vagina artificial, así mismo este método no siempre asegura una eyaculación efectiva, depende en gran medida el nivel de estimulación lograda hacia el carnero. El uso de este método ha sido prohibido en algunos países ya que se ha comprobado que el uso del electro eyaculador eleva el ritmo cardiaco y los niveles de cortisol, de tal manera Orihuela (2014) menciona que en caso de ser indispensable el uso de este método, es imprescindible asociarla con algún tratamiento anestésico para reducir los efectos estresantes en el animal.

Estados Unidos y en los lugares tropicales, la electroeyaculación aún tiene aceptación por grupos de bienestar animal, según Palmer (2005). Mientras que, en el continente europeo la electroeyaculación está contraindicadan en toros sin el uso de anestésicos, según Mosure et al., (1998)

La electroeyaculación es una técnica en la que se aplica estimulación eléctrica por medio de una sonda rectal. Este estímulo ocasiona que la musculatura de las extremidades pélvicas se contraigan, provocando también la erección del pene según Fumagalli (2012); para el uso de los impulsos nerviosos se debe manejar con sumo cuidado la intensidad de corriente eléctrica con la que se 
trabaja, debido a que no hay un voltaje exacto para el uso en todos los animales, sino que se debe ir procediendo con voltajes variados siempre de menor intensidad hacia mayo, y encontrar un voltaje óptimo para cada animal a someter a este método, debido que los animales reaccionaran de diferente manera y se tendrá que encontrar una intensidad optima por animal a tratar.

Al producir la estimulación adecuada, esta viaja vía nervio pudendo interno hacia los centros lumbosacros de la columna vertebral, desde allí parte la respuesta vía nervios simpáticos lumbares (nervio erigente del plexus hipogástrico), lo cual estimula la contracción de la musculatura lisa que recubre la próstata, glándulas vesiculares y conductos deferentes, asegurando la progresión de la masa espermática hacia la uretra pélvica (emisión). Por otro parte, la respuesta nerviosa viaja vía nervios parasimpático para provocar la contracción de la musculatura estriada del tracto uretral (músculo isquiocavernoso, bulbo esponjoso y uretral), lo cual que resulta en la erección del pene y la eyaculación propiamente dicho (Morrillo et al., 2012).

Las sondas miden aproximadamente $30 \mathrm{~cm}$ de largo y de 20 a $30 \mathrm{~mm}$ de diámetro, tienen electrodos longitudinales o transversales (Garcia-Macias, y otros, 2006); El equipo produce descargas de 1 a 15 V (Fumagalli, 2012), el cual se debera regualar su voltaje de menor intensidad a mayor para evitar lesiones o dolor en el animal hasta encontrar la intensidad adecuada para producir la eyaculación en el animal.

La xilacina es un fármaco derivado de los compuestos tiacínicos que ocasionan analgésica, sedación y relajación de los músculos. El aumento en la dosificación aumenta la duración de los efectos mas no el nivel de la sedación, que normalmente están en un promedio de $25 \pm 5$ minutos.

Dado a estas particularidades, es de mucha ayuda para sedar a animales, cuando se realizan intervenciones poco cruentos, como procedimientos radiológicos, ecográficos, curaciones, entre otros. (Sumano \& Ocampo, 2006), siendo de mucha utilidad para procedimientos que pueden ser considerados cruentos o que se ponga en riesgo el tema de bienestar animal.

\section{Metodología}

La investigación se la llevó a cabo en la granja Irquis perteneciente Universidad Estatal de Cuenca la misma que está situada en la parroquia Victoria del Portete, a una distancia aproximada de 25 km desde cantón Cuenca, provincia del Azuay siguiendo la panamericana sur, a una altitud ente los 2.500 a $3.880 \mathrm{msnm}$, con una temperatura que oscila entre $10-14^{\circ} \mathrm{C}$.

La evaluación se la hizo con 4 carneros maduros sexualmente, todos pasados el año de edad y con un margen de pesos de 60 a $90 \mathrm{Kg}$. Se sometió a un periodo de acondicionamiento a los cuatro carneros en estudio tanto de adaptación climática como en la alimentación la misma que consistió en pastoreo libre a base de Ray - grass con concentrado, sales minerales y agua a voluntad. Anticipadamente se realizó exámenes previos a los semovientes, para la determinación que estuviesen libres de cualquier tipo de enfermedades reproductivas. 
Todas las muestras sanguíneas pasaron por un proceso de centrifugación para la obtención del suero sanguíneo y la colocación en tubos eppendefor para su posterior envío de las muestras al laboratorio CENBIOCLI S.A. para la evaluación de las hormonas en estudio, en la ciudad de Cuenca.

Para la extracción de semen los 4 carneros fueron sometidos a 2 tratamientos diferentes, donde se realizó 4 repeticiones a cada uno, tratamiento 1: se extrajo contenido seminal mediante la técnica de electroeyaculación a los carneros 4 veces por cada animal, se hicieron las extracciones una vez por semana hasta obtener las 4 colectas/animal, teniendo 16 repeticiones en este tratamiento; previo a iniciar con el tratamiento 2 , se dejó descansar a los carneros por una semana; en este segundo tratamiento se utilizó xilacina en dosis de $0,05 \mathrm{mg} / \mathrm{kg}$ de peso vivo por animal, de la misma manera se procedió a realizar 4 colectas por animal una vez a la semana hasta tener un total de 16 muestras por cada tratamiento.

\section{Muestras Seminales}

Tabla 1: Cronograma de extracciones seminales

\begin{tabular}{lccccc}
\hline & Carnero 1 & Carnero 2 & Carnero 3 & Carnero 4 & Sub Total \\
\hline $\begin{array}{l}\text { Sólo electroeyaculador. } \\
\begin{array}{l}\text { Electroeyaculador con } \\
\text { aplicación de xilacina }\end{array}\end{array}$ & 4 & 4 & 4 & 4 & 16 \\
TOTAL & 8 & 4 & 4 & 4 & 16 \\
\hline
\end{tabular}

Elaborado por : Grupo de investigación.

Las extracciones de las muestras sanguíneas consistieron, para cada ovino en tres tiempos por cada extracción de la muestra seminal:

20 minutos antes de la extracción de semen con EE; inmediatamente después de la EE; 20 minutos después de la EE, a los cuales se les asignaron las siguientes nomenclaturas:

Con anterioridad se realizó la cateterización con un catlón $\mathrm{N}^{\circ} 16$ en la vena femoral, la cantidad muestra sanguínea fue de $5 \mathrm{ml}$ por cada toma, en tubos vacutainer sin EDTA, consiguientemente las muestras fueron colocadas en gradillas y posteriormente trasladadas al laboratorio de biotecnología la granja de Irquis, para la obtención del suero sanguíneo mediante centrifugación. Las muestras fueron enviadas para los análisis de testosterona y cortisol al Laboratorio Cenbiocli S.A.

\section{Análisis Hormonal}


Tabla 2: Número de muestras sanguíneas para análisis

\section{Carnero 1 Carnero 2 Carnero 3 Carnero 4 Sub Total}

\begin{tabular}{lccccc}
\hline H. Cortisol & 24 & 24 & 24 & 24 & 96 \\
H. Testosterona & 24 & 24 & 24 & 24 & 96 \\
TOTAL & 48 & 48 & 48 & 48 & $\mathbf{1 9 2}$ \\
\hline
\end{tabular}

Elaborado por : Grupo de investigación.

Para el análisis de las muestras sanguíneas se lo realizo mediante el método de ELISA para la obtención de las concentraciones hormonales.

\section{Resultados}

En los resultados obtenidos de los análisis de las muestras del suero sanguíneo de los carneros, para lo que respecta a la hormona cortisol en el procedimiento antes (10 minutos) de la aplicación del electroeyaculador, dieron con los supuesto de normalidad, según las pruebas no paramétricas de Kolmogorov - Smirnov (K - S) y la de Shapiro - Wilk (contraste de normalidad) (Tabla 4 y 5) en ambos tratamientos: con y sin tranquilizante.

Mientras que las mediciones de esta hormona durante la aplicación y después de la aplicación del electroeyaculador, cumplieron con las condiciones de normalidad solamente en el tratamiento en que se inyectó previamente xilacina, a través de las pruebas estadísticas anteriormente mencionadas. Mediante la prueba no paramétrica de Mann-Whitney (Tabla 6) se determinó que en ninguno de los casos existieron diferencias significativas entre tratamientos $(\mathrm{P}>0,05)$, por lo que las mediciones de cortisol en sangre fueron similares en los dos tratamientos (Tabla 3).

Los resultados obtenidos en la medición de la hormona testosterona en sangre antes de introducir el electro eyaculador, cumplieron con los supuestos de normalidad en el tratamiento en que no se inyectó tranquilizante (Kolmogorov - Smirnov, Tabla 4). En el caso de los valores de testosterona durante la introducción del electro eyaculador, aquellas que cumplieron con las condiciones de normalidad fueron las del tratamiento dónde no se aplicó xilacina (Pruebas de Kolmogorov Smirnov y Shapiro - Wilk, Tabla 4) y las del tratamiento en que sí se inyectó el tranquilizante (Kolmogorov - Smirnov, Tabla 5). Por otro lado, los datos correspondientes a la medida de esta hormona después de la introducción del electro eyaculador, no cumpliéndose para ninguno de los tratamientos los supuestos de normalidad.

No hubieron diferencias significativas estadísticamente por medio de la prueba de Mann - Whitney $(\mathrm{P}>0,05)$ en la Tabla 4 y 5 en la medición de testosterona entre tratamientos con la aplicación y sin aplicación de Xilacina (Tabla 3). Las diferencias significativas estuvieron marcadas en las extracciones seminales con respecto al volumen. Siendo la media para el tratamiento con Xilacina de: $0,27 \mathrm{ml}$ superior a la media del tratamiento que no se utilizó el tranquilizante. Estos valores de 
los volúmenes son bajos, pero tuvieron similitud con los que se obtuvieron en el trabajo de Marco

- Jiménez et al., (2008) cuyo estudio lo ejecutó en carneros de raza Guirra a los que aplico tranquilizante (xilacina) anticipadamente al uso del electroeyaculador, en los que obtuvieron volúmenes de 1,01 $\pm 0,1 \mathrm{ml}$. Los resultados de nuestro estudio no contrastan con los resultados de los trabajos realizados por Ledesma et al., (2014) en los cuales los resultados promedios en los volúmenes fue de 3,99 \pm 0,386 $\mathrm{ml}$ al igual en los estudios de Pineda et al., (1987), Pineda y Dooley., (1991) los mismos que colectaron muestras seminales con volúmenes grandes mediante la aplicación de la electroeyaculación.

\section{Discusión.}

Uno de los métodos más empleados en ovinos es la Electroeyaculación, proceso que produce un estrés muy marcado en los animales afectando su bienestar, por lo que para disminuir sus efectos se utilizó el tranquilizante "xilacina", evaluado en el plasma sanguíneo principalmente el cortisol junto con la testosterona, los niveles que estuvieron presentes en los resultados nos permitió medir la libido de los animales así como el estrés producido en el estudio de los aminales.

Tabla 3: Parámetros hormonales

\begin{tabular}{|c|c|c|c|}
\hline \multirow[b]{2}{*}{ Variables } & \multicolumn{3}{|c|}{ Tratamientos } \\
\hline & $\begin{array}{c}\text { Con tranquilizante } \\
\mathrm{X} \pm \text { Electroeyaculador }\end{array}$ & $\begin{array}{c}\text { Sin tranquilizante } \\
\mathrm{X} \pm \text { Electroeyaculador }\end{array}$ & Significancia \\
\hline $\begin{array}{l}\text { Cortisol Antes de la apli- } \\
\text { cación }(\mu \mathrm{g} / \mathrm{dl})\end{array}$ & $7,44 \pm 1,220$ & $5,03 \pm 0,502$ & 0,287 \\
\hline $\begin{array}{l}\text { Cortisol Durante la apli- } \\
\text { cación }(\mu \mathrm{g} / \mathrm{dl})\end{array}$ & $5,96 \pm 0,711$ & $5,04 \pm 0,357$ & 0,323 \\
\hline $\begin{array}{l}\text { Cortisol Después de la } \\
\text { aplicación }(\mu \mathrm{g} / \mathrm{dl})\end{array}$ & $4,92 \pm 0,750$ & $5,30 \pm 0,392$ & 0,254 \\
\hline $\begin{array}{l}\text { Testosterona Antes de la } \\
\text { aplicación }(\mathrm{ng} / \mathrm{ml})\end{array}$ & $2,52 \pm 0,609$ & $1,83 \pm 0,332$ & 0,696 \\
\hline $\begin{array}{c}\text { Testosterona Durante la } \\
\text { aplicación }(\mathrm{ng} / \mathrm{ml})\end{array}$ & $2,17 \pm 0,407$ & $1,69 \pm 0,237$ & 0,838 \\
\hline $\begin{array}{c}\text { Testosterona Después de } \\
\text { la aplicación }(\mathrm{ng} / \mathrm{ml})\end{array}$ & $2,22 \pm 0,562$ & $2,03 \pm 0,301$ & 0,445 \\
\hline $\mathrm{X} \pm \mathrm{EE}:$ media \pm error está & ar de la media. & & \\
\hline
\end{tabular}

Elaborado por : Grupo de investigación. 
Tabla 4: Pruebas de normalidad para el tratamiento Sin Xilacina de Kolmogorov - Smirnov y Shapiro - Wilk

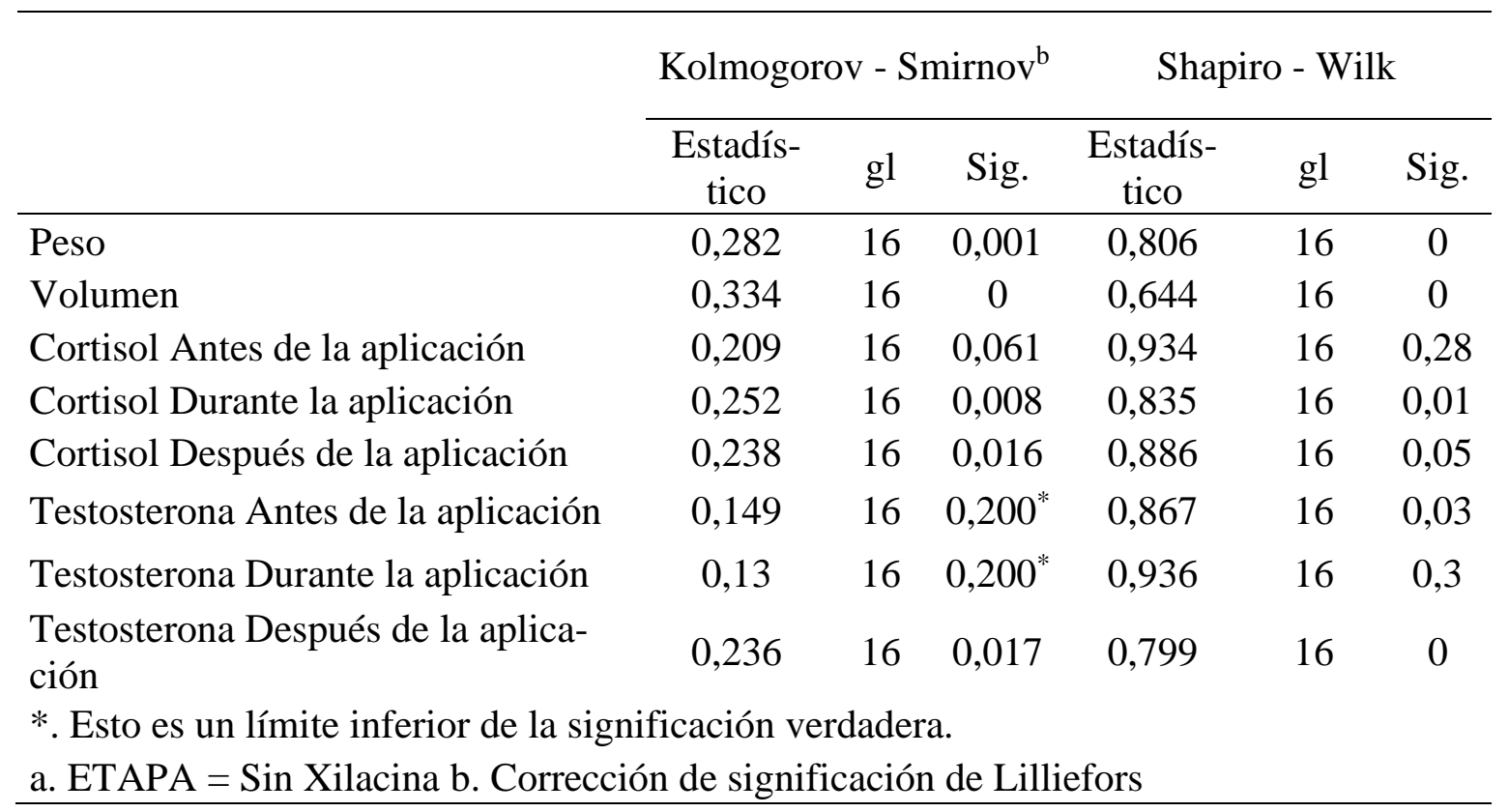

Elaborado por : Grupo de investigación.

Tabla 5: Pruebas de normalidad para el tratamiento con Xilacina de Kolmogorov Smirnov y Shapiro - Wilk

\begin{tabular}{lccccccc}
\hline & \multicolumn{3}{c}{ Kolmogorov - Smirnov ${ }^{\mathrm{b}}$} & \multicolumn{3}{c}{ Shapiro - Wilk } \\
\cline { 2 - 8 } & $\begin{array}{c}\text { Estadís- } \\
\text { tico }\end{array}$ & $\mathrm{gl}$ & Sig. & $\begin{array}{c}\text { Estadís- } \\
\text { tico }\end{array}$ & $\mathrm{gl}$ & Sig. \\
\hline Peso & 0,282 & 16 & 0,001 & 0,806 & 16 & 0 \\
Volumen & 0,197 & 16 & 0,098 & 0,87 & 16 & 0,03 \\
Cortisol Antes de la aplicación EE & 0,164 & 16 & $0,200^{*}$ & 0,909 & 16 & 0,11 \\
Cortisol Durante la aplicación EE & 0,115 & 16 & $0,200^{*}$ & 0,948 & 16 & 0,45 \\
Cortisol Después de la aplicación EE & 0,168 & 16 & $0,200^{*}$ & 0,902 & 16 & 0,09 \\
Testosterona Antes de la aplicación EE & 0,319 & 16 & 0 & 0,773 & 16 & 0 \\
Testosterona Durante la aplicación EE & 0,19 & 16 & 0,127 & 0,841 & 16 & 0,01 \\
Testosterona Después de la aplicación & 0,262 & 16 & 0,004 & 0,821 & 16 & 0,01 \\
EE & & & & & & \\
\hline
\end{tabular}

*. Esto es un límite inferior de la significación verdadera. 


\section{a. ETAPA = Con Xilacina b. Corrección de significación de Lilliefors \\ Elaborado por : Grupo de investigación.}

Elaborado por : Grupo de investigación.

Tabla 6: Prueba No Paramétrica de Mann - Whitney para el Análisis Hormonal

\begin{tabular}{lcccccc}
\hline & $\begin{array}{c}\text { Cortisol } \\
\text { Antes de } \\
\text { la aplica- } \\
\text { ción }\end{array}$ & $\begin{array}{c}\text { Cortisol Du- } \\
\text { rante la apli- } \\
\text { cación }\end{array}$ & $\begin{array}{c}\text { Cortisol } \\
\text { Después de } \\
\text { la aplicación }\end{array}$ & $\begin{array}{c}\text { Testosterona } \\
\text { Antes de la } \\
\text { aplicación }\end{array}$ & $\begin{array}{c}\text { Testosterona } \\
\text { Durante la } \\
\text { aplicación }\end{array}$ & $\begin{array}{c}\text { Testosterona } \\
\text { Después de la } \\
\text { aplicación }\end{array}$ \\
\hline U de Mann - Whitney & 99 & 101 & 97 & 117 & 122,5 & 107,5 \\
W de Wilcoxon & 235 & 237 & 233 & 253 & 258,5 & 243,5 \\
Z & $-1,093$ & $-1,018$ & $-1,168$ & $-0,415$ & $-0,207$ & $-0,773$ \\
Sig. asintótica (bilateral) & 0,274 & 0,309 & 0,243 & 0,678 & 0,836 & 0,44 \\
$\begin{array}{l}\text { Significación exacta [ } 2 *(\text { sig. } \\
\text { unilateral) ] }\end{array}$ &, $287^{\mathrm{b}}$ &, $323^{\mathrm{b}}$ &, $254^{\mathrm{b}}$ &, $696^{\mathrm{b}}$ &, $838^{\mathrm{b}}$ &, $445^{\mathrm{b}}$ \\
\hline
\end{tabular}

\section{a. Variable de agrupación: tranquilizante. b. No corregido para empates.}

Elaborado por : Grupo de investigación.

Los niveles de cortisol y testosterona en el tratamiento con xilacina coinciden con lo afirmado por Damián y Ungerfeld (2011) quienes proponen que la electro eyaculación afecta a la concentración hormonal, por lo que a medida que aumenta el cortisol, la testosterona disminuye y viceversa, después de la electro eyaculación. Según Juniewicz et al., (1987) el cortisol (corticoide) suprime la generación de GnRH a nivel hipotalámico y se suprime la secreción de LH a nivel pituitario según Matteri et al., (1984).

\section{Conclusiones.}

En relación a la medición de cortisol en los animales tratados con xilacina, no hubo una disminución del estrés en los animales en relación con el otro tratamiento donde no se los trataron con tranquilizante durante la electroeyaculación.

En cuanto a los resultados obtenidos en la medición de la testosterona; en el tratamiento donde se usó la xilacina como tranquilizante, se evidencia un efecto negativo en la concentración, esto se justifica a que el cortisol suprime la formación de la hormona liberadora de Gonadotropinas $(\mathrm{GnRH})$ a nivel hipotalámico y a su vez suprime la secreción de la hormona Luteinizante (LH) a nivel de glándula pituitaria. 
Cabe recalcar que, para la manipulación de los carneros, su respectivo manejo y aplicación del tranquilizante (xilacina), pasan por inquietud, entraban a estar en alerta y consecuentemente seguía la desesperación de los animales, por lo que se asume que los animales ya sufrían de estrés por manejo antes y durante la inducción, debido a que esta condición en lo animales no permite una buena acción del medicamento por lo tanto no se obtenía una sedación óptima.

$\mathrm{Al}$ momento de manipular a los animales, estos entraban en una etapa de angustia, debido a que los animales utilizados en el estudio no tuvieron un entrenamiento previo para la realización de la investigación.

\section{Referencias bibliográficas.}

Damián, J., \& Ungerfeld, R. (2011). The Stress Response of Frequently Electroejaculated Rams to Electroejaculation: Hormonal, Physiological, Biochemical, Haematological and Behavioural Parameters. Reprod Dom Anim 46, 646-650.

Fumagalli. (2012). Parámetros fisiológicos y bioquímicos durante la electroeyaculación bajo anestesia general en el venado de campo. Uuguay: Tesis de maestría en Reproducción Animal. Recuperado el 123dok, de https://mx.123dok.com/document/w7q0gxz6parametros-fisiologicos-durante-la-electroeyaculacion-bajo-anestesia-en-venado-decampo-ozotoceros-bezoarticus.html

Garcia-Macias, V., Martinez-Pastor, F., Alvarez, M., Garde, J., Anel, E., Anel, L., \& Paz, P. (2006). Assessment of chromatin status (SCSA) in epididymal andejaculated sperm in Iberian red deer, ram and domestic dog. Theriogenology 66, 1927-1930.

uniewicz, P., johnson, B., \& Bolt, D. (1987). Effect of adrenal steroids on testosterone and luteinizing hormone secretion in the ram. Journal of Andrology vol 8, 190-196.

Metteri, R., Watson, J., \& Moberg, G. (1984). Stress or acute adrenocorticotrophin treatment suppresses LHRH-induced LH release in the ram. Journal of Reproduction and Fertility 72, 385-392.

Morrillo, M., Salazar, S., \& Castillo, E. (2012). Evaluación del potencial reproductivo del macho bovino. Instituto de Investigaciones Agrícolas, 23-28.

Mosure, W., Mayer, R., Gudmundson, J., \& Barth, A. (1998). Evaluation of possible methods to reduce pain associated tith electroejaculation in bulls. Can Vet, 504-506.

Orihuela, A. (2014). La conducta sexual del carnero. Rev Mex Cienc Pecu, 49-89. 
Palmer, C. W. (2005). Welfare aspects of theriogenology : Investigatingalternatives to electroejaculation of bulls. ELSEVIER, 469-479

Palmer, C., Brito, L., Soderquist, A. A., Persson, Y., \& Barth, A. (2005). Comparison of electroejaculation and transrectal massage for semen collection in range and yearling feedlot beef bulls. ELSEVIER, 25-31. 
PARA CITAR EL ARTÍCULO INDEXADO.

Ulloa Ramones, L. A., \& Ulloa Ramones, D. A. (2020). Evaluación de los niveles de cortisol y testosterona durante el proceso de colecta de semen mediante electroeyaculador en carneros con y sin tranquilizante. Ciencia Digital, 4(2), 65-76. https://doi.org/10.33262/cienciadigital.v4i2.1206

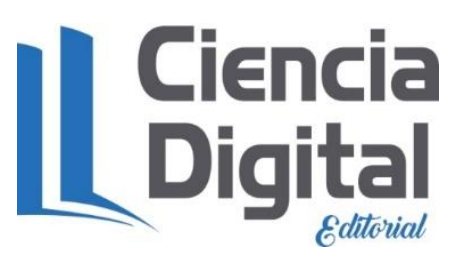

El artículo que se publica es de exclusiva responsabilidad de los autores y no necesariamente reflejan el pensamiento de la Revista Ciencia Digital.

El artículo queda en propiedad de la revista y, por tanto, su publicación parcial y/o total en otro medio tiene que ser autorizado por el director de la Revista Ciencia Digital.
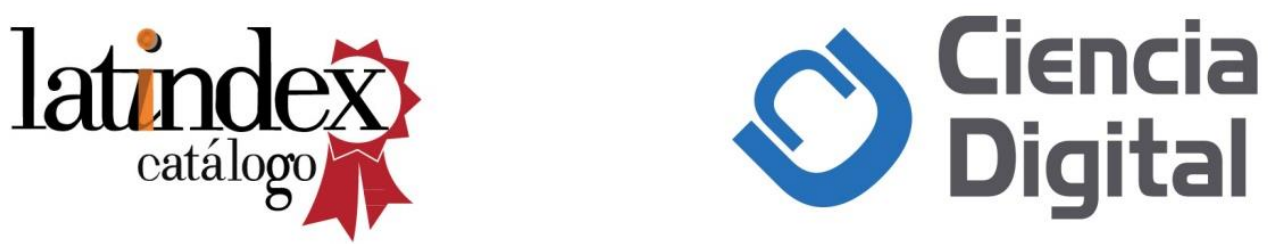